\title{
Measuring Potential from Muscle Activity of Lower Arm
}

\author{
S. Herwiningsih, S. P. Sakti, F. Yuana
}

\begin{abstract}
Muscle is one of body tissues which is excitable. It poses an electrical activity during contraction and relaxation which could be measured using an electrical device called as electroneuromyograph (ENMG). This device has a wide application in neurophysiology, biomechanics, fatigue analysis, and ergonomic, as well as sports. However, there is a limited access to this device for research due to its scarce availability which is mainly found in certain hospitals in urban area causing an expensive cost to perform the test. This paper presents a work in measuring biopotential produced from activity of lower arm muscles using a simple ENMG module. An instrumentation amplifier was used to amplify signals from the muscle which are detected using three surface electrodes connected to the amplifier using an EMG cable. The output signal was displayed using an oscilloscope. The results show that the average value of the peak-to-peak voltage during contraction and relaxation were $460 \mathrm{mV}$ and $32.6 \mathrm{mV}$, respectively. This indicates that a simple ENMG module can be used for measurement the electrical activity of the muscle tissues. The measured signal fluctuated during the measurement, which is possibly caused by the arm position during measurement, the attachment of the surface electrode on the skin surface and the body movement during measurement.
\end{abstract}

Keywords: muscle electrical activity, electroneuromyograph, biopotential, muscle contraction.

\section{INTRODUCTION}

Movement is one of the vital functions of the human body which involves the works of muscular and skeletal systems, as well as nervous system. Muscle tissues are excitable, enabling the muscle to contract and relax. The stimulus send by the nervous tissue triggers the action potential that fires the muscle tissues and propagates along the muscle fibers. The electrical potential in the muscle tissues arises from the difference concentration of ions in the extracellular and intracellular fluids which is separated by a selective permeable cell membrane. The electrical activities of the muscle could be recorded using electroneuromyography (ENMG).

ENMG plays an important role in medicine, especially to detect abnormalities in the muscle and neuromuscular

Revised Manuscript Received on January 5, 2020.

* Correspondence Author Natural Science, Universitas Brawijaya, Malang, Indonesia.

Email: herwin@ub.ac.id

S. P. Sakti, Department of Physics, Faculty of Mathematics and Natural Science, Universitas Brawijaya, Malang, Indonesia. Email: sakti@ub.ac.id Science, Universitas Brawijaya, Malang, Indonesia. Email: firdy@ub.ac.id
Sri Herwiningsih*, Department of Physics, Faculty of Mathematics and

F. Yuana, Department of Physics, Faculty of Mathematics and Natural

junction. This device is useful in evaluating the neurophysiological condition of the motoric nerves, peripheral nerves, and the muscle function [1]. It has been shown that ENMG plays role in detecting the muscle activity in the patients with chronic respiratory disease [2], evaluating the respiration pattern of newborn baby, predicting the lung function of the children with asthma [3], detecting speaking ability in deaf people [4], and fatigue analysis [5]. The ENMG is not only a powerful tool for study the muscle function in human, but also in animals [6].

In principle, ENMG measures the potential of the muscle by placing two electrodes in both ends of the muscle and connected with the oscilloscope. When muscle is excited, the action potential propagates from motoric nerves to muscle. This action potential then propagates along the muscle fibers and producing the contraction of the muscle.

Different types of electrodes are usually used in the measurement of ENMG signal, which are needle electrode and surface electrode. The needle electrodes offers a better sensitivity than the surface electrodes, however it might cause discomfort to the object (patient) due to the insertion of the needle during the measurement. Although surface electrode is less sensitive and only measure the electrical activity of superficial muscles, it is preferable by the patients as it does not cause pain during measurement. The major issues with the measurement of signal produced from the body is the signal is relatively weak. It has been reported that the signal produced from the muscle usually ranges from $0-10 \mathrm{mV}$ and frequency of $20-500 \mathrm{~Hz}$ [7]. Therefore it requires the instrumentation amplifier to enable signal recording.

Although ENMG has a wide application, the use of ENMG is still limited due to the limited access [3], especially in the developing countries such as in Indonesia. The availability of ENMG is limited in the big hospitals in urban area, causing an expensive cost to perform the test. This causes difficulty in accessing this device for research in the field of biomechanics and neurophysiology as it is mainly used for medical services. This work aims to explore the ability of a simple ENMG to measure the potential produced from the muscle activity. It is expected that from the simple ENMG, it could be further developed for exploration of static and dynamic of human movement and ergonomics.

\section{MATERIALS AND METHODS}

This work was divided into two parts. The first part was construction of a simple ENMG module. For this

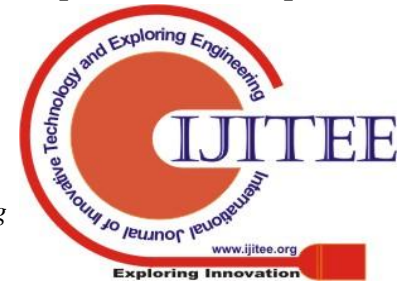


purpose, IC AD8226 was used as an instrumentation amplifier to amplify the signal recorded from the muscle activities. The amplified signal was then rectified using a rectifying circuit and passing through the smoothing circuit to obtain a rectified-smoothed signal.

The module was powered using two $9 \mathrm{~V}$ batteries. To detect the signal from the muscle, three surface electrodes were used, and the output signal was displayed using digital oscilloscope (GWInstek GDS-1102-U).

The second part was testing the module. The amplification of the ENMG module was tested by using an input voltage of 8 Volt and input frequency of $10 \mathrm{KHz}$ produced from the signal generator. The output voltage after passing the ENMG module was recorded. The gain of the amplifier could be adjusted by rotating the variable resistor. The potential gain was calculated by dividing the output potential with the input potential.

The ENMG module was then used to measure the biopotential produced from the activity of lower arm muscle. Three surface electrodes were used for this purpose by attaching the electrodes on the skin surfaces. Before electrode attachment, the skin was cleaned up using water to ensure that the electrode attached tightly to the skin surface. The first electrode was placed at the end of the muscle (about $5 \mathrm{~cm}$ from the elbow joint), the second electrode was placed in the middle part of the muscle (about $5 \mathrm{~cm}$ from the first electrode) and the last electrode was placed in the bony part of the arm closed to the elbow joint as the reference. The attached electrodes were connected to the ENMG module using EMG cables. The output signal was observed using the digital oscilloscope. The measurement was performed when the muscle relax, i.e. the palm open and when the muscle contract, i.e. the palm squeeze. The measurement was repeated three times.

\section{RESULTS AND DISCUSSION}

The result of amplification testing of the ENMG module was presented in Table-I. It shows that the average value of the amplification of the voltage (potential) of the ENMG modules is about three times. The amplification of the module was adjusted by rotating the variable resistor. Rotating the resistor variable half way results in the attenuation of the potential of 0.07 times. It shows that the amplification of the ENMG module is relatively small. Although the variable resistor has been varied, the highest amplification obtained was only three times.

Table- I: Testing of amplification of the ENMG module

\begin{tabular}{|c|c|}
\hline Input Voltage (V) & Output Voltage (V) \\
\hline 8 & 24.4 \\
\hline 8 & 22.8 \\
\hline 8 & 28.8 \\
\hline 8 & 20.8 \\
\hline Average & 24.2 \\
\hline
\end{tabular}

The results of the measurement of lower arm muscle activity are presented in Table-II. The results show that the average value of peak-to-peak voltage ( $\mathrm{Vpp}$ ) during muscle contraction is $460 \mathrm{mV}$. While the average value of $\mathrm{Vpp}$ during muscle relaxation is $32.6 \mathrm{mV}$. This indicates that during muscle relaxation, the measured potential is smaller compared to the measured potential during muscle contraction. During muscle contraction, the action potential was propagated along the muscle fiber, producing a higher potential.

Table- II: Biopotential measurement of the lower arm muscle

\begin{tabular}{ccc}
\hline Measurement & $\begin{array}{c}\text { Vpp during } \\
\text { muscle } \\
\text { contraction }(\mathbf{m V})\end{array}$ & $\begin{array}{c}\text { Vpp during } \\
\text { muscle } \\
\text { relaxation }(\mathbf{m V})\end{array}$ \\
\hline 1 & 340 & 30 \\
2 & 720 & 36 \\
3 & 320 & 32 \\
Average & 460 & 32.6 \\
\hline
\end{tabular}

The visual display of the measured signal during the muscle contraction and relaxation are shown in Fig. 1 and Fig. 2, as recorded by the digital oscilloscope. In both figures, the vertical scale of the recorded signal was $200 \mathrm{mV} / \mathrm{div}$.

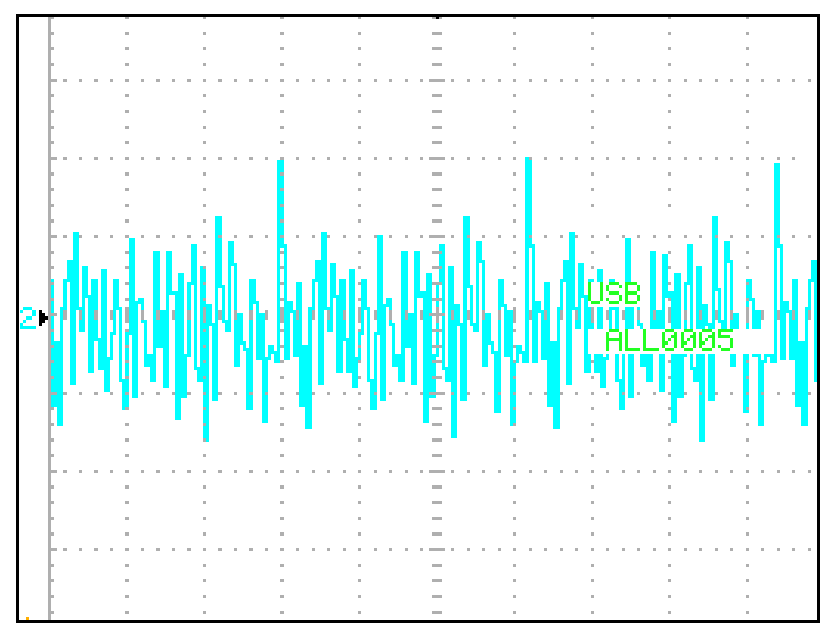

Fig. 1. The recorded signal during muscle contraction

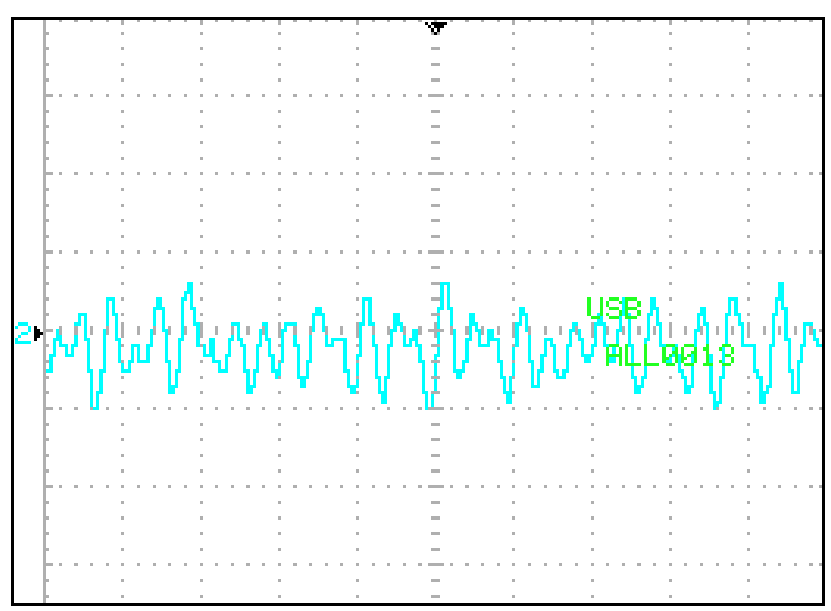

Fig. 2. The recorded signal during muscle relaxation

Although the measured biopotential from the lower arm muscle is relatively small, the results are still within the reported value in literature, i.e. 0.4 to 5 Volt. Sumanto et al. [8] who measured the 
activity of biceps brachii muscle (upper arm) reported the biopotential of 0.5 Volt during relaxation and 3.8 Volt during muscle contraction. Another work by Multajam et al. (2016) reported the measured biopotential of 2.25 Volt when the palm squeezed, and 1.6 Volt when the palm opened [9].

The smaller value obtained from this work possibly due to the lower amplification used from the instrumentation amplifier. This causes the detected signal also lower compared to the reported by other studies $[8,9]$.

In addition, we found that the measured biopotential fluctuates during measurement. It is possibly caused by several factors. The first possible factor is the position of the arm during measurement. It is reported that the arm positioned on the sides of the body during measurement results in a higher signal (2.64 Volt), compared to the biopotential measured when the arm is folded 90 degree (1.6 Volt). In this both situations, the arm muscle are relax [9]. This indicates that the position of the arm might affect the measured biopotential from the arm muscle. The next possible factor that affecting the measured biopotential is the attachment of the surface electrode. The measured signal would be high enough when the electrode is attached tightly on the surface of the skin. The reused surface electrode would not produce a better signal, rather than a weak signal as the electrode does not attached tightly compared to its first use. Therefore, it is highly recommended to change the surface electrode every time the new measurement is performed. The third possible factor that affect the measured signal is the movement of the body during the measurement. A little movement causes instability on the recorded signal. This is possibly caused by the use of lengthy EMG cables that connect the electrodes and the ENMG module, in which the movement of the body would move the cable, causing signal fluctuation.

\section{CONCLUSIONS}

It has been shown that a simple electroneuromyograph could be used to detect the electrical activity of the muscle of lower arm. The simple electroneuromyograph consists of an instrumental amplifier, three surface electrodes and oscilloscope for displaying the output signal. The measurement of the muscle potential from lower arm shows that the average potential value during the muscle contraction was $460 \mathrm{mV}$, while during the relaxation was $32.6 \mathrm{mV}$. The measured signal was affected by several factors: the position of arm during measurement, the attachment of the surface electrode on the skin surface and the movement of the body during measurement. Future works would focus on improving the performance of the instrumental amplifier, in order to produce stronger signals from the measured muscle.

\section{ACKNOWLEDGMENT}

The author would like to thanks LPPM Universitas Brawijaya for assisting this project. The author also thanks Triswantoro Putro, and Nabila Ratna Ayuningtyas for their valuable help during completion of the work.

\section{REFERENCES}

1. A. R. Sonkaya, \& M. Karaoğlan, (2019), Investigation of concordance between referral diagnosis and electroneuromyographic diagnosis, J. Surg. Med., Vol. 3 (3), 2019, pp. 250-243, doi:10.28982/josam.519328.

2. I. M. M. Dos Reis, D. G. Ohara, L. B. Januário, R. P. Basso-Vanelli, A. B. Oliveira, \& M. Jamami, Surface electromyography in inspiratory muscles in adults and elderly individuals: A systematic review, Journal of Electromyography and Kinesiology, Vol. 44, 2019, pp. 139-155. Available: https://doi.org/10.1016/j.jelekin.2019.01.002.

3. G. J. Hutten, H. F. van Thuijl, A. C. M. van Bellegem, L. A. Eykern, \& W M. C. van Aalderen, A literature review of the methodology of EMG recordings of the diaphragm, Journal of Electromyography and Kinesiology, Vol. 20 (2), 2010, pp. 185-190. Available: https://doi.org/10.1016/j.jelekin.2009.02.008

4. E. R. Handiarno, M. Sarwoko, R. Purnamasari, Construction of Electronyograph for Speech Detection of Vocal Alphabet in Deaf Person, E-Proceeding of Engineering, Vol. 2 (3), 2016, pp. 6980-6987. (In Indonesian)

5. J. G. A. Cashaback, T. Cluff, \& J. R. Potvin, Muscle fatigue and contraction intensity modulates the complexity of surface electromyography, Journal of Electromyography and Kinesiology, Vol. 23 (1), 2013, pp. 78-83. Available: https://doi.org/10.1016/j.jelekin.2012.08.004

6. S. Valentin, \& R. R. Zsoldos, Surface electromyography in animal biomechanics: A systematic review, Journal of Electromyography and Kinesiology, Vol. 28, 2016, pp. 167-183. Available: https://doi.org/10.1016/j.jelekin.2015.12.005

7. D. Arifianto, W. Winarno, \& R. Apsari, Construction of Monitoring System and Data Logging of Electromyograph Signal Using Wireless, 'Presented at APTIKOM National Seminars', Mataram, Indonesia, 2016. (In Indonesian)

8. B. Sumanto, A. M. Handayani, \& M. Rinardi, Construction of Electronic Module for Detection of Electromyogram Signal, 'Presented at National Seminars of Applied Technology SV UGM', Yogyakarta, Indonesia, 2015. (In Indonesian)

9. R. Multajam, M. Sanjaya, A. Sambas, N. Subkhi, \& I. Muttaqien, Design and Analysis of Electromyography (EMG) and its Application in Detecting Muscle Signals, ALHAZEN Journal of Physics, Vol. 2 (2), 2015, pp. 38-48. (In Indonesian)

\section{AUTHORS PROFILE}

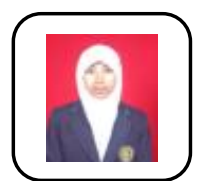

Sri Herwiningsih, BSc (Physics), M.App.Sc (Medical Physics), Ph.D (Physics), her research interest is in Biophysics and Medical Physics fields.

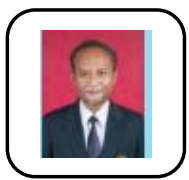

S. P. Sakti, BSc (Physics), M. Eng (Electronic Engineering), Dr.-Ing (Sensor and Microsystem), his research interest is in Sensor Engineering.

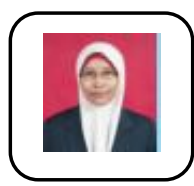

F. Yuana, BSc (Physics), M.Sc (Medical Physics), her research interest in Biophysics and Medical Physics. 